\title{
La muerte de Daniel Alcides Carrión: una revisión crítica
}

\section{Daniel Alcides Carrion's death: a critical review}

\author{
David Salinas-Flores ${ }^{1,2}$ \\ ${ }^{1}$ Médico, Departamento de Medicina Hospital Alberto Sabogal. Lima, Perú. \\ ${ }^{2}$ Profesor de la Facultad de Medicina, Universidad Nacional Mayor de San Marcos. Lima, Perú.
}

"La ciencia histórica, como ocurre en general con todas las ciencias, no es nunca final" Sir Powicke M. Moderns Historians and the study of historie. 1955.

\begin{abstract}
Resumen
El experimento de Daniel Alcides Carrión marcó un hito en la historia de la medicina peruana. Alrededor de él ha existido mucha controversia. Quizás, el principal tema de debate sea la causa de su muerte. En la controversia, ha pasado desapercibido un hecho objetivo ocurrido en las últimas horas del experimento: la administración de inyecciones endovenosas de ácido fénico. Esta fue una terapia propuesta en Perú en aquella época como tratamiento del carbunco. Los médicos de la junta médica, en la agonía de Carrión, probablemente no administraron la transfusión sanguínea a Carrión, para experimentar con este tratamiento. La junta médica pensaba quizás repetir con la bartonelosis el éxito descrito que tuvieron las inyecciones de ácido fénico con el carbunco. Desconocían la actualmente demostrada toxicidad de esta sustancia. Probablemente, el evento precipitante de la muerte de Carrión fue una intoxicación por ácido fénico en un paciente con bartonelosis en fase anémica.

Palabras clave: Perú, historia de medicina; carbunco; Carrión, Daniel A; bartonelosis; fenol.
\end{abstract}

\begin{abstract}
Daniel Alcides Carrion's experiment was a landmark in the history of Peruvian medicine, with much controversy, but perhaps the main topic of discussion is the cause of Carrion's death. An objective fact has passed unnoticed in the last hours of the experiment: the administration of intravenous injections of phenic acid. In 1884, this was a therapy proposed for anthrax treatment in Peru. The doctors' medical decision during Carrion's agony probably did not consider giving blood transfusions to Carrion in order to experiment with this new treatment. They probably thought to repeat with bartonellosis the success described with phenic acid injections in a patient with anthrax, unaware of the now proven toxicity of phenic acid. The event precipitating Carrion's death was probably phenic acid intoxication in a patient with bartonellosis in the anemic phase.
\end{abstract}

Key words: Peru, history of medicine; anthrax; Carrion, Daniel A; Bartonella Infections; phenol.

\section{Introducción}

El experimento de Daniel Alcides Carrión y los conocimientos extraídos acerca de él representan el segundo aporte más importante de la medicina peruana, siendo el primero la quinina. El interés por continuar con el legado de Carrión hizo que la bartonelosis sea la enfermedad más investigada en toda la historia de la medicina peruana ${ }^{(1)}$. Aunque el estudio de la enfermedad ha seguido una línea ascendente, los estudios históricos sobre el experimento de Carrión han sido distorsionados, impidiendo una correcta interpretación ${ }^{(2)}$. Alrededor de su sacrificio ha existido mucha controversia. Quizás, el principal tema de debate sea la causa de la muerte de Carrión. Es probable que la respuesta la encontremos en un análisis del contexto y los documentos de la época.

\section{El experimento de \\ Daniel Alcides Carrión}

Carrión era un interno de medicina del Hospital San Bartolomé, pero eligió el servicio de su profesor Leonardo Villar, del recientemente inaugurado Hospital Dos de Mayo, porque era el que más pacientes con verrugas tenía. La inoculación fue realizada en presencia de algunos compañeros suyos, como Julián Arce, quien años más tarde fundaría la cátedra de Medicina Tropical en la Facultad de Medicina de la Universidad de San Marcos. Salvo una escueta nota en el Monitor Médico y en El Comercio dando cuenta de la inoculación, no hubo algún otro pronunciamiento por los médicos locales.

El móvil que llevó a Carrión a realizar su experiencia fue el deseo de conocer el inicio de la enfermedad de la verruga. Para él era muy importante conocer los síntomas, por lo que no quiso experimentar con animales ${ }^{(3)}$. Él quería aprender a diagnosticar tempranamente la verruga peruana, quizás para tratarla con mayor éxito. Así lo dijo el mismo Carrión y así fue trascrito por sus compañeros que lo asistieron, en la obra que publicaron el año siguiente ${ }^{(4-7)}$. Sin embargo, durante el transcurso del experimento, Carrión al ver el período febril que se instalaba en él, sin habérselo propuesto inicialmente, comprueba una hipótesis formulada anteriormente por otros investigadores: que la Fiebre de la Oroya y la verruga peruana son la misma enfermedad ${ }^{(8)}$. Los resul- tados de tan valerosa experiencia fueron una sorpresa para todos, incluso para el propio experimentador ${ }^{(3)}$, un acto que algunos investigadores han considerado como un ejemplo de serendipia ${ }^{(9)}$.

No se ha encontrado hasta la actualidad alguna evidencia del proyecto o plan de investigación de Daniel Alcides Carrión para realizar su experimento ${ }^{(1)}$. Tan solo existe una descripción de este: "El 27 de agosto de 1885, a las 10 h, Daniel Alcides Carrión, alumno del $6^{\circ}$ año de la Facultad de Medicina de San Fernando, logró (no sin dificultad) que su amigo, el Dr. Evaristo M. Chávez, le practicara cuatro inoculaciones, dos en cada brazo, cerca del sitio en donde se hace la vacunación. Dichas inoculaciones se hicieron con la sangre inmediatamente extraída por rasgadura de un tumor verrucoso del enfermo Carmen Paredes, de la sala Nuestra Señora de las Mercedes del Hospital Dos de Mayo, perteneciente al servicio del Dr. Villar" (4).

A los veintitrés días de realizada la inoculación, se manifestaron los primeros síntomas. Luego de la inoculación, Carrión solo fue asistido por algunos de sus compañeros provincianos de clase. 
Sus profesores no lo asistieron, ni les interesó científica o caritativamente. Fue visitado por sus profesores solo en su agonía. Cuando él murió, sus prestigiosos profesores salvaron su responsabilidad al declarar solemnemente que la inoculación se hizo desautorizadamente. Aceptó ser visitado por su profesor el doctor José María Romero, quien prescribió quinina por si se tratara de malaria, sin resultado. Evolucionó peor, con calambres, dolor abdominal, náuseas y vómitos. El 2 de octubre de 1885 , se encontraba en mal estado general, adelgazado y deshidratado, realizándose en ese momento la primera junta médica. El día 3 de octubre, el Dr. Ricardo Flores le realiza un recuento globular, el primero efectuado en el Perú, recomendándole una transfusión sanguínea. El 4 de octubre es evaluado por una segunda junta médica, conformada por los doctores Villar, Romero, Flórez y Chávez. Carrión manifestó su deseo de ser trasladado a la clínica Maison de Santé para que se realizara la transfusión sanguínea. El Dr. Villar había llevado un transfusor de Oré para proceder a la operación y uno de los condiscípulos daría las onzas de sangre. Fue conducido a la clínica francesa porque era el único lugar que contaba con el aparato para realizarle la operación de transfusión, como se le llamaba en esa época ${ }^{(10)}$. En la clínica ocurrió un hecho inesperado y hasta ahora no explicado: la transfusión sanguínea no se realizó.

El diario del experimento de Carrión dice: "Preocupado (Carrión) con el resultado de la junta que en esos momentos acababa de reunirse pregunta a los que les rodean si ya estaba resuelta la transfusión, que en su opinión era la única tabla salvadora que le quedaba. Grande fue su contrariedad y desaliento cuando supo que la consulta había dado por resultado aplazar la operación, tanto más cuanto, según decía, era el único móvil que tuvo para resolverse a abandonar una casa donde hubiera preferido concluir sus días" (4).

\section{La última medicina de Carrión: un remedio contra el carbunco}

El documento redactado por los compañeros de Carrión menciona: "No obstante la opinión de la mayoría de la junta médica a favor de la trasfusión sanguínea, esta fue aplazada para el día siguiente. Este hecho sorprendió al propio Carrión, porque él había abandonado su domicilio con ese objetivo. En lugar de la transfusión queda sometido al siguiente tratamiento: inyecciones intravenosas de ácido fénico y 20 centigramos de albuminado de fierro cada dos horas; se continuaron además las inhalaciones de oxígeno y las pulverizaciones de ácido fénico" (4). Esa fue la última medicación que recibió Carrión. Su estado se siguió deteriorando hasta que falleció a las 11:30 de la noche del 5 de octubre de $1885^{(4,10,11)}$.

Para muchos, siguen siendo desconocidas las razones por las cuales no se administró a Carrión una transfusión para subvenir la severa anemia del proceso, aunque parte de la explicación pueda deberse a la inseguridad de las transfusiones sanguíneas ${ }^{(11,12)}$, dado que los grupos sanguíneos recién fueron descubiertos en 1900. La razón más probable es que se esperaba el éxito de las inyecciones de ácido fénico, una terapia propuesta por otro profesor de Carrión, el profesor Tomas Salazar, un año antes.

El Dr. Tomás Salazar (1831- 1917), profesor de Terapéutica y Materia Médica de la Facultad de Medicina de San Fernando, fue un producto directo y ejemplar de la reforma de la educación médica que realizó Cayetano Heredia. Bajo la dirección del propio Heredia, realizó el primer trabajo científico sobre bartonelosis. Su tesis fue traducida al inglés y reproducida por varios diarios de Londres (13). Según Odriozola, fue el primer estudio clínico verdaderamente serio sobre esta enfermedad ${ }^{(14)}$. No solo fue el primero en publicar un trabajo de bartonelosis, sino que 27 años antes que Carrión fue el primero en postular que la Fiebre de la Oroya y la verruga peruana eran la misma enfermedad. Sin embargo, aunque el Dr. Salazar es conocido en la ciencia peruana por su investigación de la enfermedad de Carrión, años después, como profesor de terapéutica realizaría un trabajo en su área con otra enfermedad infecciosa. En 1884, publicaba en La Crónica Médica su trabajo: "Pústula maligna, curación por medio de inyecciones intravenosas de ácido fénico" (15). Pústula maligna era el nombre con el que se conocía en el siglo XIX al carbunco o ántrax. Su trabajo se basaba en que el Bacillus anthracis circulaba en la sangre y, por ende, la terapia debería ser administrada por vía sanguínea. En el trabajo describe un paciente que tuvo una buena evolución con este tratamiento. La elección del ácido fénico no fue al azar; era la sustancia química que Lister usó para iniciar la antisepsia. Era previsible que años más tarde algún médico intentara usar esta sustancia no solo a nivel local, sino a nivel sistémico. Era probablemente la primera vez que se aplicaba una terapia sistémica en el tratamiento del carbunco. Hasta esa fecha, el tratamiento era a nivel tópico. Durante el siglo XIX, los conocimientos sobre esta enfermedad estaban en un nivel muy avanzado. El problema principal era su tratamiento. Las primeras ediciones de los tratados internacionales de medicina interna recomendaban el tratamiento tópico, a base de quemaduras con formol, electrocauterio o ácidos. Esta realidad también ocurría en el Perú. Algunas tesis peruanas de medicina humana así lo demuestran. Sin embargo, Salazar planteaba un tratamiento diferente: la vía sistémica ${ }^{(16)}$. La investigación de Salazar transcendió a nivel internacional; mereció citaciones a nivel mundial en los principales indexadores médicos del siglo XIX: la revista médica francesa Revue bibliographique Universelle des Sciences Medicales, publicación en la que se revisaba los principales avances a nivel mundial de la época (precursor del Medline actual), y The Bristol Medico-chirurgical Journal ${ }^{(17)}$. Se consideraba hasta esa fecha como un aporte de la ciencia peruana el uso de inyecciones endovenosas para el tratamiento del carbunco. Este hecho es resaltado por otro prolífico investigador médico peruano del siglo XIX, Leonidas Avendaño, fundador de la principal revista médica peruana del siglo XIX La Crónica Médica, quien en un artículo publicado el mismo año del experimento de Carrión, en dicha revista escribió: "Antes de concluir esta parte quiero dejar constancia de que en ninguno de los casos curados por las inyecciones neutralizantes de sustancias antivirulentas que se han publicado, se han llevado el agente antiséptico directamente al torrente circulatorio. El método de inyecciones intravenosas en el tratamiento de las enfermedades carbunculosas es exclusivamente peruano... y en el año pasado de 1884 nuestro antiguo 
catedrático de Patología Médica, el Doctor Tomas Salazar, sin tener conocimiento de los hechos anteriores y guiado únicamente por el convencimiento que tenía de poder atacar el Bacillus anthracis en la sangre, empleó las inyecciones intravenosas de ácido fénico en un caso de pústula maligna que progresaba a pesar de la cauterización, obteniendo una curación completa" ${ }^{(18)}$.

El impacto nacional e internacional que tuvo este experimento terapéutico de Salazar probablemente sea la explicación de por qué la junta médica que atendió a Carrión decidió aplazar la transfusión sanguínea y esperar el éxito de las inyecciones de ácido fénico como una terapia contra la verruga, en sus últimas horas. Luego de haber fracasado la quinina, era el único descubrimiento peruano hasta esa época.

Es necesario enfatizar que el experimento de Carrión fue realizado un año después de la publicación del experimento terapéutico del profesor Salazar, en sus pacientes con carbunco. La junta médica probablemente pensaba repetir con la bartonelosis el éxito descrito que tuvieron las inyecciones de ácido fénico con el carbunco.

El experimento de Daniel Alcides Carrión tuvo así como contexto la investigación peruana del carbunco. Ambas investigaciones interactuaron: la investigación de Salazar sobre el carbunco influyó en la muerte de Carrión y la investigación de Carrión opacó la investigación peruana sobre carbunco que se realizaba a fines del siglo XIX. Tomás Salazar, con su trabajo sobre bartonelosis, que probablemente le sirvió de modelo a Carrión, influyó en la génesis de su experimento. Pero además, con su investigación del carbunco con el ácido fénico, influyó también en el final de su experimento.

El experimento de Daniel Alcides Carrión tuvo un contexto científico mundial caracterizado por la era de los descubrimientos de los agentes infecciosos; del nacimiento de la infectología, que fue iniciada por la investigación pionera del carbunco de Koch y Pasteur. Por otro lado, las inyecciones de ácido fénico administradas a Carrión, en sus últimas horas, aunque fueron dadas como una medida salvadora, probablemente no dejaron de ser observadas también como un experimento científico, dado que este tratamiento no había sido empleado antes. El propio Carrión, en sus apuntes menciona que el tratamiento de la verruga peruana tiene dos objetivos principales: $1^{\circ}$ favorecer la erupción por medio de infusión de uña de gato y $2^{\circ}$ combatir por los medios más activos la anemia ${ }^{(4)}$. No existía, hasta la experiencia de Carrión, comunicaciones sobre el uso de ácido fénico endovenoso para tratar la bartonelosis. Era, de acuerdo a todas las evidencias, un tratamiento experimental.

Aunque la inoculación de Carrión se realizó en presencia de sus profesores, después de esta, él fue abandonado a su propia suerte; solo fue atendido por sus propios compañeros de estudios. Sin embargo, en sus últimas horas se revela un interés de los profesores, no solo asistencial sino probablemente también científico, como lo demuestra un incidente ocurrido inmediatamente después de la muerte de Carrión, que ha pasado desapercibido durante estos años de estudio sobre este tema. Según se describe en la necropsia de Carrión: "Finalmente, la vena mediana cefálica del brazo derecho había sido abierta post mortem para procurarse sangre con qué hacer experiencias en animales inferiores" ${ }^{(19)}$. Así, se realizó una extracción de sangre del recién fallecido Carrión, para luego realizar la inoculación con su sangre de dos conejos, la cual dio resultados fallidos, en palabras de un investigador de Carrión: “A quién en un momento tan dramático como era la muerte del joven estudiante, se le ocurriría continuar con la observación experimental? ¿Quién dispuso de su ejecución?" ${ }^{(1)}$. El experimento diseñado por Carrión con fines clínicos se transformó, probablemente por la junta médica, en un inesperado experimento terapéutico; es decir, experimentar una terapia del carbunco en la bartonelosis.

El uso del ácido fénico como sustancia endovenosa para el tratamiento de las infecciones no fue un caso aislado. Existió en el Perú, a fines del siglo XIX, posteriormente al experimento de Salazar, una búsqueda de sustancias que podrían haber sido 'los antibióticos del siglo XIX' que eran conocidos como 'los específicos', sustancias contra una determinada enfermedad infecciosa. Si Salazar preconizaba el ácido fénico endovenoso para tratar el carbunco, un año después Leonidas Avendaño, otro profesor de Carrión, en asociación con el Dr. Nicolás Corpancho, experimentaron con amoníaco endovenoso en pacientes con carbunco. Solo tres semanas después de la muerte de Carrión, el 28 de octubre de 1885, Leonidas Avendaño escribía en la Crónica Médica "Si mis previsiones resultan verdaderas... le quedará a la medicina peruana la gloria de haber iniciado y establecido cuatro medicaciones por las inyecciones intravenosas: el cloral para el tétano (Dr. Lino Alarco), el amoníaco para las infección purulenta (Dr. Lino Alarco), el cápsicum para la fiebre amarilla (Dr. Armando Vélez y FP del Barco) y el amoníaco para el carbúnculo (Dr. Néstor Corpancho y Leonidas Avendaño)" (18).

Probablemente, los profesores de Carrión esperaban que la quinta enfermedad que la medicina peruana hubiera iniciado tratamiento por medio de inyecciones intravenosas, iba a ser la bartonelosis por medio del ácido fénico. En los años siguientes, los médicos peruanos conocerían los efectos tóxicos del ácido fénico cuando es absorbido a nivel sistémico, como es reflejada en una tesis de la época (20) y probablemente también de otras sustancias que eran experimentadas por esa época, por vía sistémica; por lo que estas terapias endovenosas no fueron utilizadas por mucho tiempo en el Perú. El ácido fénico, aunque inicialmente se utilizó como un antiséptico orgánico, debido a su toxicidad actualmente se emplea como anestésico local y antipruriginoso ${ }^{(21)}$. En 1910, Julián Arce escribiría que lo ideal sería descubrir un 'agente terapéutico específico', como por ejemplo la quinina para la malaria, y él preconizó el uso del salvarsán para la bartonelosis ${ }^{(22)}$, que fue empleado hasta la llegada de los antibióticos, dado que ya en 1944 se publicó el primer caso de bartonelosis tratado exitosamente con penicilina ${ }^{(23,24)}$.

\section{La muerte de Carrión}

Basado en el antecedente de inoculación con sangre de un paciente con verruga, la pirexia extrema y la anemia hemolítica severa que Carrión desarrolló, es muy probable que haya tenido bartonelosis. En 1898, Odriozola afirmaba: "El fenómeno 
de la anemia, que consideramos como el elemento clínico culminante de la fiebre grave de Carrión, es en nuestro concepto el signo fundamental de su identidad, pues no hay enfermedad alguna con la que pueda compararse, sobre todo por la rapidez de su desarrollo" (14). El sulfato de quinina que se le administró fue ineficaz , por lo que se puede afirmar que su enfermedad no fue paludismo ${ }^{(25)}$.

Algunos investigadores han propuesto la exhumación de los restos de Carrión para que, con las actuales técnicas de secuenciación molecular de ADN, documentar de modo más objetivo la bartonelosis, dado que al momento de su muerte, la sangre médula ósea y vísceras debieron haber estado densamente impregnados del agente patógeno de la enfermedad: la Bartonella bacilliformis ${ }^{(26)}$.

Según algunos investigadores, la principal interrogante del experimento de Carrión es: ¿De qué murió Carrión? (5). El informe oficial de los médicos legistas que realizaron la autopsia de Carrión dice: "Que a consecuencia de la operación (inoculación), enfermó y murió el operado con la fiebre de verrugas el 5 del presente a las once de la noche" (19). Según el historiador Lastres y Carlos Monge: "La enfermedad que mató a Carrión fue la verruga" ${ }^{(6,27)}$. Sin embargo, Carrión nunca afirmó que moría de Fiebre de la Oroya ${ }^{(28)}$. La bartonelosis habría llevado a Carrión a un estado de sepsis con anemia severa. La decisión de haberse formado dos juntas médicas demuestra la gravedad del estado de Carrión. Sin embargo, aunque la anemia y la septicemia hubieran debilitado la resistencia de su organismo, estas no explican por completo su muerte. La anemia, aunque severa en Carrión (1 085 000/mL de glóbulos rojos), no era necesariamente mortal; supervivencias de anemias severas de apenas medio millón de glóbulos rojos/mL han sido registradas ${ }^{(29)}$. Dada la ausencia de pérdida de sangre, la anemia era probablemente de tipo hemolítico.

En la muerte de Carrión, la controversia se ha limitado a si él falleció a consecuencia de la bartonelosis pura o de una infección secundaria concomitante, de naturaleza salmonelósica ${ }^{(29,30)}$. Sin embargo, se ha ignorado un hecho más objetivo ocurrido en las últimas horas, que fueron las inyecciones de acido fénico. Aunque algunos investigadores han considerado controversial hasta qué punto fueron tóxicas las inyecciones de ácido fénico ${ }^{(31)}$, su toxicidad ha sido demostrada ${ }^{(32)}$.

Los hallazgos de la necropsia no pueden ser usados para investigar con solidez signos de toxicidad por ácido fénico, dado que la autopsia de Carrión, que fue realizada en medio de numerosos médicos y estudiantes de la Facultad de Medicina, solo revelaba el precario conocimiento de la época en relación a patología ${ }^{(33)}$. No se hizo exámenes toxicológicos ni anatomopatológicos; fue una escena de confusión ${ }^{(34)}$. En 1898, en una tesis de San Marcos, se afirmaba: "No debe olvidarse que uno de los mejores síntomas de la intoxicación fenicada es el color negro que toma la orina" ${ }^{20}$. Esta información sigue vigente en la actualidad ${ }^{(35)}$. Lamentablemente, en el folleto publicado por los compañeros de Carrión no hubo mayores registros médicos después de la administración de inyecciones de ácido fénico, como las características de la orina, que podrían documentar mejor los efectos del ácido fénico en Carrión.

La autopsia ha sido definida desde el punto de vista de medicina legal como el conjunto de investigaciones que se realiza sobre el cadáver, encaminadas al estudio de las causas de la muerte ${ }^{(36)}$. Aún en famosas autopsias de recientes personajes históricos, es difícil lograr una autopsia completa. Sin embargo, debe cumplir el objetivo, que es determinar la causa de la muerte ${ }^{(37)}$. En la autopsia de Carrión solo se constató marcada palidez, disminución de la grasa corporal, hepatomegalia, adenomegalia mesentérica y un bazo de pequeño volumen ${ }^{(4)}$. La autopsia no fue adecuada, dado que estos datos de la autopsia no permiten determinar por sí solos la causa de la muerte de Carrión.

En la actualidad, el ácido fénico tiene solo aplicación tópica, no sistémica, por lo que los pocos casos de intoxicación por ácido fénico no son endovenosos. Sin embargo, existen evidencias históricas de su uso sistémico, como en el experimento de Carrión. Así, pese al fracaso de las inyecciones de ácido fénico en salvar a Carrión, varios médicos en el Perú continuaron imitando el uso que se hizo en aquella oportunidad, empleando inyecciones de ácido fénico para casos avanzados de la Fiebre de la Oroya, como sucedía en el Hospital Militar de San Bartolomé, lugar donde Carrión hacía su internado y que era también el lugar donde Tomas Salazar, creador de este método, trabajaba y donde administraba su terapia con inyecciones de ácido fénico. A solo un mes de la muerte de Carrión, Enrique Mestanza, probablemente el compañero más cercano a Carrión, dado que hacían internado en el mismo hospital y quien fue testigo de su agonía, empleaba inyecciones de ácido fénico en el servicio de Salazar, en pacientes con bartonelosis, sin el éxito esperado. La dosis empleada era 1 gramo de ácido fénico en 80 gotas de agua destilada; de esta solución, se inyectaba dos tercios en la vena safena ${ }^{(38)}$. Irónicamente, otro compañero de Carrión, Julián Arce, cuatro años después de la muerte de Carrión, en 1889, criticaba esta terapia en su tesis de graduación como bachiller en medicina, donde menciona: "Creemos que el tratamiento más inaceptable y más peligroso que puede oponerse a la fiebre de la Oroya en la actualidad es el de los antisépticos y sobre todo el de las inyecciones intravenosas de soluciones de ácido fénico, usados por algunos de nuestros prácticos.... No creo pues, que por ahora debamos dar la preferencia a ese tratamiento, no veo la razón para tal preferencia" ${ }^{(39)}$. Y basado probablemente en la experiencia de Carrión, en la que la transfusión sanguínea fue aplazada, Julián Arce menciona: "Si lejos de mejorar se agrava el estado del enfermo, debe sin demora practicarse la transfusión sanguínea y no esperar tímidamente hasta el último momento" (figura).

Pero, probablemente la demostración más contundente de la toxicidad del ácido fénico, también conocido como fenol, cuando es aplicado por medio de inyecciones endovenosas, haya ocurrido durante la Segunda Guerra Mundial. Los nazis, en los campos de exterminio de Auschwitiz, usaban inyecciones de acido fénico endovenosas para ultimar a sus víctimas, en sus famosos experimentos médicos; el método llegaría a convertirse en el método más común de asesinato individual. El médico nazi Joseph Mengele, en sus experimentos con gemelos, ordenaba asesinar a los niños con inyecciones 


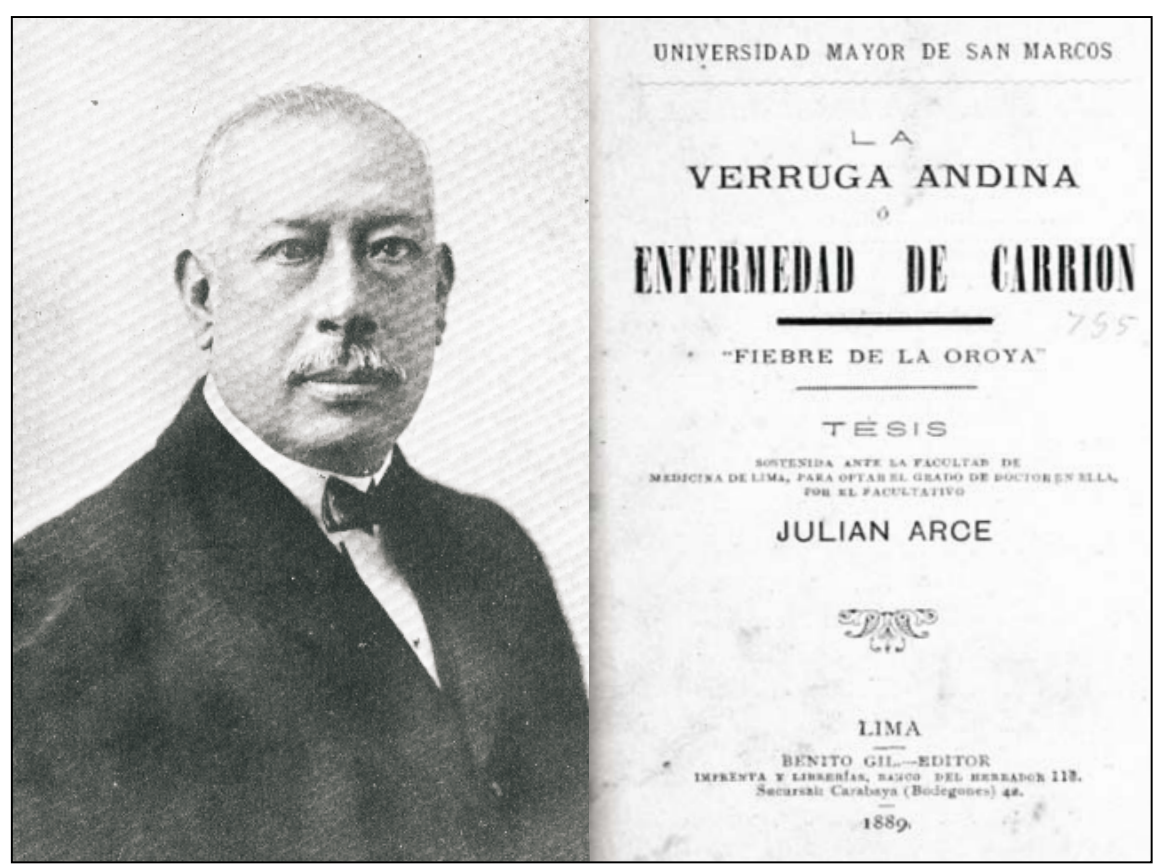

Figura. Dr. Julian Arce, foto extraída de Valdizán H. Diccionario de Medicina Peruana Tomo II. Tesis Doctoral Arce J. La Verruga andina o enfermedad de Carrión. UNMSM 1889.

de ácido fénico, para luego analizar sus órganos comparativamente en la autopsia. Un caso conocido de víctimas de los nazis por inyecciones de ácido fénico es el caso del sacerdote polaco Kolbe, quien fue canonizado por el papa Juan Pablo II, en $1982^{(40,41)}$.

Basado en esta evidencia, se puede afirmar que probablemente la muerte de Carrión fue precipitada por una intoxicación medicamentosa por ácido fénico, en un paciente que de base tenía sepsis y anemia severa por bartonelosis. Las inyecciones de ácido fénico fueron solo un factor precipitante; el destino fatal de Carrión era inevitable, pues la sepsis y la anemia severa llevarían a la muerte a Carrión en un tiempo probablemente mayor. La transfusión sanguínea solo le hubiera servido de modo muy restringido, dado que el $50 \%$ de hematíes inyectados de una persona sana a una enferma son parasitados y destruidos prematuramente ${ }^{(42)}$. Así, la transfusión solo hubiera prolongado por un tiempo su agonía. Los antibióticos, la única terapia salvadora, aún no habían sido descubiertos.

Es necesario destacar que la junta médica tuvo una visión pionera para emplear la vía sistémica para tratar la enfermedad de Carrión, que es la vía terapéutica que se emplea en la actualidad. Sin embargo, en esa época aún no existían los antibióticos. Los médicos de la junta pensaron que el ácido fénico podía haber sido su equivalente. Lamentablemente, el descubrimiento de los antibióticos y su llegada al Perú ocurriría recién 50 años después del experimento de Carrión. El método pionero de la junta era el correcto, la sustancia empleada era equivocada; así, probablemente el evento precipitante de la muerte de Carrión fue una iatrogenia médica, una intoxicación por ácido fénico, en un paciente con bartonelosis en fase anémica.

En contraste con la autoinoculación que pasó casi inadvertida, la muerte de Carrión causó gran revuelo. Las principales revistas científicas nacionales y mundiales se dedicaron al tema. Un año después, en 1886, el mundo se enteraría de las nueve historias clínicas que recopiló Carrión cuando sus compañeros de estudios editaron sus apuntes en un folleto, el cual fue publicado por resolución ministerial por el Estado Peruano. Mariano Alcedán, compañero de estudios de Carrión, propuso, en 1886, en su honor, el nombre de Enfermedad de Carrión.

Por otro lado, la subprefectura en Intendencia de Policía ordenó una in- vestigación completa de los hechos, por considerar que el acto equivalía a "suicidio u homicidio calificado condenado por nuestras leyes" (7). Sin embargo, la investigación policial solo indagó el evento de la inoculación del agente infeccioso. No se investigó la terapia experimental con ácido fénico endovenoso.

La investigación sobre la Fiebre de la Oroya -nombre equivocado, porque la enfermedad no existió en la Oroya, el nombre se dio por el destino final del tren- continuó, en 1909. El Dr. Alberto Barton descubrió el agente etiológico de la verruga. En 1915, el Dr. Richard Strong la denomina Bartonella bacilliformis. Hasta 1993, la única especie descubierta del género Bartonella fue la Bartonella bacilliformis; pero, actualmente el género Bartonella incluye a 18 especies, de las cuales ocho son patógenas para el hombre ${ }^{(43)}$. Así, la bartonelosis, que era considerada como una enfermedad exótica y una curiosidad científica médica de algunas regiones de Sudamérica, es considerada hoy como una de las principales enfermedades emergentes. Desde 1997, se ha registrado la aparición de nuevas zonas endémicas de la enfermedad de Carrión en zonas de selva alta y en zonas de la sierra, como en el valle sagrado de los incas, en Cusco ${ }^{(44-50)}$, lo que ha llevado a que un siglo después las autoridades de salud hayan realizado nuevamente una convocatoria para investigar la enfermedad de Carrión. Así, esta enfermedad, que debería quedar en la historia, aún tiene vigencia en el Perú debido al subdesarrollo ${ }^{(51)}$.

Daniel Alcides Carrión, a ciento veinticuatro años de su muerte, es probablemente el personaje más importante de la medicina peruana y con alcance universal. La gran mayoría de los historiadores de la medicina nacional ha ensalzado a Carrión empleando adjetivos superlativos, como protocientífico, positivista, entre otros ${ }^{(5)}$. Su imagen facial fue inicialmente 'europeizada'. Otros consideran su experimento como un acto arriesgado de un 'serrano emergente' ${ }^{(33)}$. Carrión ha llegado a ser graduado póstumamente como médico y doctor en el Perú (52). Mucho se ha dicho y se ha escrito sobre su vida y la importancia de su legado. El presente trabajo pretende analizar el contexto científico en relación a su muerte, de 
un modo objetivo, como probablemente Carrión lo hubiera querido.

\section{REFERENCIAS BIBLIOGRÁFICAS}

1. Murillo JP, Salaverry O, Mendoza W, Franco G, Calderón W, Rodríguez-Tafur J. Daniel Alcides Carrión y su contribución al imaginario cultural de la Medicina Peruana. An Fac med. 2002;63:141 59.

2. García Cáceres U. La fascinación por Daniel A. Carrión. Actualidad Médica. 1990;2:5.

3. Arce J Lecciones sobre verruga peruana 0 enfermedad de Carrión. An Fac med. 1918;1:33.

4. Medina C, Mestanza E, Arce J, Alcedán M, Miranda R, Montero M. La Verruga Peruana y Daniel A. Carrión (1886). 3ra Ed. Lima: Cuerpo Médico del Instituto Sanitas; 1957.

5. Pamo RO. Daniel Carrión: Mito y realidad. Rev Med Hered. 2003;14:214-20.

6. Monge C. La enfermedad y la muerte de Carrión. An Fac med. 1925;8:86-91.

7. Leonard J. Daniel Carrion and Carrion Disease. Bull Pan Am Health Organ. 1991;25:3.

8. Cueto M. Tropical medicine and bacteriology in Boston and Peru: studies of Carrion's disease in the early twentieth century. Med Hist. 1996;40:34464.

9. Stella A. La contribución de Carrión: un ejemplo de serendipia. Folia Derm Peru. 2001;3:63-7.

10. Perales A. Evaluaciónética dela autoexperimentación de Daniel A Carrión y su perfil de personalidad. An Fac med. 2003;64:180-98.

11. Graña A. Daniel A. Carrión: heroísmo y controversia. Acta Med Per. 2007;24:245-8.

12. Benites JM. De la Transfusión Sanguínea, Tesis de Bachiller N ${ }^{0} 24$, Facultad de Medicina, Universidad Nacional Mayor de San Marcos. 1884.

13. García Cáceres U. Cayetano Heredia: Un símbolo. Acta Herediana. 1991;2:22-33.

14. Odriozola E. La maladie de Carrión ou la verruga peruvienne. Paris: Carré y Naud; 1898.

15. Salazar T. Pústula maligna, curación por medio de inyecciones intravenosas de ácido fénico. La Crónica Médica. 1884;1:132.

16. Salinas-Flores D. Ántrax en Perú: La investigación pionera del siglo XIX. Rev Soc Per Med Int. 2001;14:44-9.

17. Gali M. Sociedad Unión Fernandina Sección Variedades. La Crónica Médica. 1885;2:295.

18. Avendaño L. Las enfermedades carbunculosas y su específico: El amoníaco. La Crónica Médica. 1885;2:411-26.

19. La Puente I, Loli V, Vega M. Documentos oficiales relativos a DA Carrión. Sociedad Unión Fernandina Sección Oficial. La Crónica Médica. 1885;2:402.
20. Pardo Figueroa E. Accidentes debido al empleo del ácido fénico como antiséptico en cirugía. Tesis doctoral. UNMSM. 1898.

21. Litter M. Farmacología Experimental y Clínica. 7a Ed. Lima: Editorial El Ateneo; 1986:14-6.

22. Arce J. Lecciones sobre la verruga peruana "Enfermedad de Carrión." An Fac Med. 1918;2:4952.

23. Merino C. La penicilina en el tratamiento de la Enfermedad de Carrión. La Gaceta Médica. 1944;1:28.

24. Gorbitz G. Experiencias terapeúticas en verruga peruana. Lima: Ed Rimac; 1948.

25. García-Cáceres U. El auténtico Daniel Alcides Carrión. El Comercio. 9 octubre 1998.

26. Arias Stella J. Propuesta al Colegio Médico del Perú. Discurso de orden en la Celebración del Día de la Medicina Peruana y Homenaje al Héroe nacional Daniel Alcides Carrion. Diagnóstico. 2007:46:214-6.

27. Lastres. Daniel Alcides Carrión. Lima: Edit. UNMSM; 1957. p. 67.

28. Urteaga BO. Verruga Peruana o Enfermedad de Carrión. Arch Per Patol Clin. 1967;21:107-36.

29. Cuadra M, Cuadra AL. Enfermedad de Carrión: inoculaciones de seres humanos con Bartonella bacilliformis, una revisión. An Fac med. 2000;61:289-94.

30. Deza B, Herrera C, Salinas C, Un documento esclarecedor acerca de la causa de la muerte de Daniel A Carrion. Revista del IPSS. 1992;11:512.

31. Pamo R. Daniel Carrion a 100 años de su muerte. En: Temas de la historia médica del Perú. Lima: CONCYTEC; 1990:40.

32. Gupta S, Ashrit G, Chandra D, Gupta AK, Finkel KW, Guntupalli JS. Acute phenol poisoning: a life-threatening hazard of chronic pain relief. Clin Toxicol (Phila). 2008;46:250-3.

33. García Cáceres U. Historia Crítica de Daniel A. Carrión y de la Medicina de su Época. Tesis Doctoral. Universidad Peruana Cayetano Heredia. Lima, Perú. 1971.

34. Shultz G. Daniel Carrion's experiment. N Engl J Med. 1968;278:1325.

35. Balcells. La Clínica y el Laboratorio. Barcelona: Edit Masson; 2002:4.

36. Rodríguez A, Verdú P. La autopsia histórica: presentación del método y su aplicación al estudio de un hecho violento ocurrido en Uruguay en el año 1972. Rev Med Uruguay. 2003;19:126-39.

37. Lundberg GD. Closing the case in JAMA on the John F. Kennedy autopsy. JAMA. 1992;268:1738.

38. Mestanza C. Revistas Clínicas. Hospital San Bartolomé. Servicio del Dr. Tomas Salazar. El Monitor Médico. 1885;1:263.
39. Arce J. La Verruga Peruana o Enfermedad de Carrión. Tesis de bachiller en Medicina. UNMSM. Lima, Perú. 1889.

40. Lifton, RJ. Killing with syringes: phenol injections. En: The Nazi Doctors: Medical Killing and the Psychology of Genocide. New York: Basic Books; 1986.

41. Alexander L. Medical science under dictatorship. N Engl J Med. 1949;241:41-2.

42. Reynafarje C, Ramos C. The hemolytic anemia of human bartonelosis. Blood. 1961;17:574.

43. Maguiña C. Las viejas y nuevas bartonellas: una nueva enfermedad emergente mundial. Acta Med Per. 2001;18:122.

44. Maguiña C, Ugarte-Gil C y col. Actualización de la enfermedad de Carrión. Rev Med Hered. 2008;19:36-41.

45. Montoya M, Maguiña C, Vigo B, y col. Bartonelosis en el Valle Sagrado de los Incas (Cusco). Bol Soc Perú Med Int. 1998;11:170-6.

46. Arias Stella J. Bartonellosis: de enfermedad regional a infección emergente mundial. Acta Méd Per. 1999;1:43-54.

47. Espejo K. 9 millones en riesgo por mal de Carrión. La República. 23 set 2006.

48. Verruga peruana causa una muerte en Lima. Perú 21. 19 set 2006.

49. Cruz V, Vargas C. Bartonelosis aguda complicada. Presentación de 44 casos, Huancabamba. Piura. Rev Soc Per Med Interna. 2003;16:5-9.

50. Burstein Z, Mayta P. En defensa de la denominación como "Enfermedad de Carrion" para la bartonelosis humana producida por la bartonella bacilliforme Rev Per Med Exp Salud Pública. 2007;2:103-6.

51. Ministerio de Salud. Contratación de consultoría para desarrollar un programa de investigación en bartonellosis con impacto en salud pública. Instituto Nacional de Salud. Lima: Ministerio de Salud; 2007.

52. Delgado G. Daniel Alcides Carrión, mártir de la medicina peruana, héroe nacional. Asociación de Historia de la Medicina Peruana. Lima: Fondo Editorial de la Universidad Nacional Mayor de San Marcos; 2001.

Manuscrito recibido el 15 de abril de 2009 y aceptado para publicación el 20 de mayo de 2009.

Correspondencia:

Dr. David Salinas Flores

Federico Villlarreal 592 Urb. Ingenieria

San Martin de Porres

Lima 31, Perú

Correo-e: dsalinas2009@yahoo.com 